\title{
La ruralité contemporaine face aux changements climatiques : étude de l'adaptation sur les littoraux touristiques d'une petite ville québécoise
}

\author{
Alexis Guillemard ${ }^{a}$, Dominic Lapointe ${ }^{b}$
}

RÉSUMÉ. En études urbaines ou régionales, les petites et moyennes villes (PMV) forment une catégorie difficile à classer. En périphérie, ces territoires hybrides comprennent des signes de l'urbain, mais aussi certaines fonctions associées à la ruralité (p. ex., agriculture et conservation). Le tourisme y est souvent désigné comme une solution au recul agricole et à la désindustrialisation. Pourtant, en situation littorale, le secteur touristique des PMV dépend d'un environnement fragile, soumis à divers événements naturels amplifiés par les changements climatiques. Au Québec, les littoraux de l'estuaire du Saint-Laurent font face à plusieurs pressions liées aux transformations du climat: accélération de l'érosion côtière, diminution du pied de glace, incertitudes autour des tempêtes, submersion, etc. C'est notamment le cas à Rivière-du-Loup, petite ville du BasSaint-Laurent. À l'aide de la théorie de la production de l'espace (Lefebvre, 1974/2000), nous essayons de mettre en lumière les principaux enjeux pour l'adaptation du territoire touristique louperivois. Nous nous basons sur certains résultats préliminaires d'une recherche-action en mode living lab que nous avons menée de 2016 à 2019.

ABSTRACT. In urban or regional studies, small and medium-sized cities (SMC) are a specific urban category. In the periphery, these hybrid territories include signs of the urban, but also certain activities associated with rurality (agriculture and conservation, for example). Tourism is often referred to as a solution to agricultural decline and deindustrialization. However, in coastal areas, the tourism sector in the SMCs depends on a fragile environment that is subject to various natural hazards amplified by climate change. In Quebec, the coasts of the St. Lawrence Estuary face several pressures linked to climate change: accelerated coastal erosion, reduced ice-foot, uncertainties with storms, submersion, etc. This is particularly the case in Rivière-du-Loup, a small city in the Bas-Saint-Laurent region. Using the theory of production of space (Lefebure, 1974 (2000), we try to highlight the main issues to adapt the Rivière-du-Loup tourist region. Our research is based on some preliminary results of an action-research in living lab mode that we conducted between 2016 and 2019.

\section{Introduction}

Que cela soit en études urbaines ou régionales, les petites et moyennes villes (PMV) constituent des territoires à part. En plus de leurs fonctions urbaines, les enjeux agricoles et de conservation des milieux naturels y occupent une place importante. Cette hybridité inscrit les PMV des régions périphériques parmi les diverses formes de la ruralité contemporaine. Les acteurs touristiques intègrent donc cette pluralité dans leurs plans de développement. Aux côtés des attractions culturelles, la nature et l'agriculture y occupent une place importante, ce qui implique une plus grande dépendance au climat que dans les métropoles. En effet, dans le système touristique d'une métropole, l'attractivité nait surtout de la concentration et du rayonnement des ressources culturelles (Gagnon, 2003) ainsi que du poids du tourisme d'affaires (Pilette et Kadri, 2005; Kadri et Pilette, 2017). Les acteurs de l'urbanisme et du tourisme s'allient pour concentrer

\footnotetext{
a Doctorant, Département études urbaines et touristiques, ESG-UQAM; Groupe de recherche et d'intervention tourisme territoire et société

b Ph. D., professeur, Département études urbaines et touristiques, ESG-UQAM; Responsable du Groupe de recherche et d'intervention tourisme territoire et société
} 
les attraits dans un périmètre relativement réduit et uniformisé : la bulle touristique (Judd, 1999).

De leur côté, les petites villes ne bénéficiant pas d'un produit d'appel très fort doivent composer leur offre touristique avec des attraits éclatés sur le territoire. Par rapport au tourisme urbain des grandes villes, le tourisme des PMV dépend donc d'un assemblage qui inclut leur terroir, leurs attraits naturels, leurs écosystèmes, leurs paysages et quelques attraits culturels. L'arrière-pays joue donc un rôle considérable dans leur développement touristique. Les changements climatiques sont susceptibles de transformer ces systèmes touristiques dépendant de la nature et du climat, particulièrement dans les milieux désignés comme vulnérables. Or, au Québec, les littoraux du Saint-Laurent font partie des territoires les plus sensibles aux évolutions du climat (Ouranos, 2015). Dans cette recherche, nous étudions une petite ville côtière du Bas-SaintLaurent : Rivière-du-Loup. Malgré une population de près de 20000 habitants (Statistique Canada, 2016a), Rivière-du-Loup peut être définie comme petite sur le continuum des PMV car, hormis le cégep, elle ne concentre pas de services d'échelles régionales (p. ex., hôpital, université, pôle industriel, institutions de direction régionale, etc.).

En 2016, la MRC de Rivière-du-Loup compte 33958 habitants (Statistique Canada, 2016b), dont 17973 à Rivière-du-Loup. Les autres municipalités qui nous intéressent dans la MRC se situent sur le littoral: Notre-Dame-du-Portage, Cacouna et L'Isle-Verte (ainsi que l'île de Notre-Dame-desSept-Douleurs). Nous nous intéressons à l'échelle de la MRC car ce découpage institutionnel donne une échelle d'analyse pertinente pour analyser le système touristique louperivois, qui dépasse les frontières de la ville. En outre, les municipalités qui entourent Rivière-du-Loup forment une agglomération sous son influence (migrations pendulaires, magasinage, centre d'activités sportives et culturelles, etc.). Réciproquement, les municipalités limitrophes donnent aux Louperivois des lieux d'excursion et des accès à l'eau intéressants. Dans la MRC de Rivière-du-Loup, des usages de conservation coexistent avec des fonctions agraires et urbaines. Ici, la ruralité s'échappe du cadre campagnard agricole et symbolise bien la diversification des formes rurales et leurs rapports à l'urbain.
Cette ruralité contemporaine se reflète notamment dans la mutation économique des régions périphériques. Le tourisme s'y développe pour pallier les difficultés des secteurs primaire et secondaire. Dans notre cas, des lieux aux usages multiples (loisirs, chasse, pêche, conservation, agriculture, etc.) sont mis en valeur à des fins touristiques dans la baie de Notre-Dame-du-Portage, au parc côtier Kiskotuk ou encore à L'Isle-Verte.

En face du territoire de la MRC, l'archipel d'îles sous la gestion de la Société Duvetnor occupe une place à part puisque sa fonction touristique sert à la protection de ce sanctuaire. Le fleuve Saint-Laurent constitue le support et la trame de fond qui unissent ces différents attraits. Cependant, l'estuaire se transforme, sous les effets des changements climatiques.

Nous voulons comprendre comment les acteurs touristiques locaux s'approprient localement cet enjeu actuel, souvent déterritorialisé par des discours globalisants. C'est à l'aide de la théorie de la production de l'espace de Lefebvre (1974/2000) que nous aborderons la question de l'adaptation aux changements climatiques des espaces touristiques littoraux, en nous penchant particulièrement sur le cas de la MRC de Rivière-du-Loup.

\section{La production d'espaces touristiques dans les petites villes : évolution de la ruralité et vulnérabilité aux changements climatiques}

\subsection{La production de l'espace}

La théorie de la production de l'espace occupe une place incontournable parmi les legs du philosophe et sociologue Henri Lefebvre. Cette théorie nait dans le contexte d'une rationalisation à outrance de l'aménagement du territoire et de l'urbanisme français, dans les années 1960-1970. Son auteur attaque le surdécoupage et le manque de profondeur des analyses de l'espace, dont « les recherches aboutissent soit à des descriptions [...], soit à des fragmentations et découpages de l'espace» (Lefebvre, 1974/2000, p. 13).

Pour aller plus loin et pour affiner les études spatiales, Lefebvre met en avant l'existence de la relation dialectique entre la production et l'espace. Dans une société industrialisée où l'espace social 
s'étend et où l'espace naturel se réduit, l'espace est façonné par et pour la production. Dans un mouvement dialectique, la production ne peut, elle aussi, se dissocier de l'espace. La production de l'espace peut être utilisée pour interpréter l'émergence du tourisme en périphérie.

Lefebvre mentionne à plusieurs reprises le tourisme dans sa théorie. Il en fait un marqueur des transformations des campagnes françaises. En effet, pour lui, la croissance du secteur symbolise la fin des ruralités «traditionnelles» (organisées autour de l'agriculture et de la nature) et la tertiarisation des sociétés occidentales. Très critique, il dénonce le tourisme des "planificateurs » comme une parade pour les espaces en crise et une diversion à la chute des modèles agricoles occidentaux. Malgré le mépris que le développement du tourisme lui inspire, Lefebvre soulève plusieurs points importants dans la compréhension de l'espace touristique et dans la production de la destination.

Le premier point qui nous intéresse est la triplicité de l'espace. Ce processus, divisé en trois parties dialectiques, exprime comment l'espace est produit. Ainsi se superposent et s'entre-influencent l'espace conçu, l'espace vécu et l'espace perçu (Lefebvre, 1974/2000). Cette idée peut être opérationnalisée pour déconstruire la création d'un attrait touristique :

1. Espace conçu: Des acteurs publics et privés conçoivent un espace à visiter. Il s'agit de l'espace conçu, issu des représentations de l'espace des acteurs qui cherchent à le rendre efficace et cohérent;

\section{Espace vécu: Toutefois, cet espace rationalisé} est soumis à l'influence constante des représentations qui suscitent l'attractivité d'un lieu. Il s'agit de l'espace vécu des représentations et des symboles;

3. Espace perçu: Enfin, par la pratique, par le déplacement physique et par le voyage sensitif, le visiteur et le visité perçoivent l'espace, se l'approprient. C'est l'espace perçu par les pratiques spatiales, qui peut remodeler les représentations de l'espace (espace conçu) et l'espace des représentations (espace vécu).

L'utilisation de la triplicité de la production de l'espace de Lefebvre pour l'appliquer aux espaces touristiques permet de rendre compte de la totalité du tourisme et de nuancer l'idée répandue du tourisme comme remède miracle aux inégalités de développement. En effet, l'approche traditionnelle du tourisme revient à le considérer comme un secteur industriel et un enjeu économique.

Dans cette perspective, l'espace touristique est conçu et son développement est le résultat d'une stratégie rationnelle. Or, si une mise en marché efficace est nécessaire, des facteurs culturels difficiles à contrôler expliquent également la production de l'attractivité touristique. Ainsi, la transformation d'un lieu en attrait naît de l'adhésion collective à des spécificités territoriales par les visiteurs, mais aussi par les populations locales. L'approche de l'espace vécu appliquée au tourisme rend compte de la complexité inhérente à ce secteur : concevoir un espace pour les touristes ne suffit pas à en assurer le succès. De plus, l'espace perçu différencie et hiérarchise la mise en tourisme des territoires. Les enjeux d'accessibilité, de proximité et de localisation hiérarchisent les territoires, et s'avèrent parfois des obstacles infranchissables.

Certains projets touristiques québécois pas encore aboutis reflètent la multidimensionnalité de la production d'un attrait. Par exemple, malgré les moyens considérables investis dans la Cité de l'énergie de Shawinigan, elle peine encore à s'imposer comme un incontournable du circuit touristique québécois. En Gaspésie, le village de Percé est érigé comme un des symboles du Québec. Ainsi, le célèbre rocher Percé (un îlot rocheux à la forme inhabituelle) apparait dans beaucoup de campagnes de promotion à l'étranger de la destination Québec. Ce territoire dépend donc du tourisme et le rythme de l'activité économique se calque sur la saisonnalité touristique. Or, le village reçoit peu de visiteurs par rapport au volume de touristes qui visitent la province. La distance aux grands centres récepteurs et émetteurs de touristes ainsi que la raréfaction des moyens de transport pour y accéder constituent des problèmes trop contraignants pour être compensés par une notoriété importante - malgré un espace symboliquement fort.

Ainsi, la production d'espaces touristiques dans les petites villes en périphérie ne cadre pas dans les catégories construites par les approches touristiques traditionnelles. Le tourisme qui s'y pratique ne correspond pas à la définition du tourisme urbain, dans un 
contexte où les attraits culturels de centre-ville ne suffisent pas à convaincre beaucoup de visiteurs. On ne peut pas non plus parler de tourisme de nature puisqu'il existe tout de même un centre qui rassemble les caractères de l'urbanité. Enfin, l'agrotourisme se développe dans certains lieux, mais n'est pas une voie adaptée à tous les territoires.

\subsection{L'attractivité des PMV : une vulnéraibilité accrue aux changements climatiques?}

Le tourisme fait donc partie des secteurs économiques souvent choisis par les petites villes en périphérie (Pumain, 1999; Jamot, 2003). Certaines d'entre elles disposent d'avantages comparatifs : des attraits pour les visiteurs.

L'attractivité d'un site explique son développement touristique (Gagnon, 2003). Cette attractivité est une construction sociale des sociétés émettrices et réceptrices. Plusieurs approches existent pour détailler les éléments qui justifient l'intérêt de visiteurs. En premier lieu, l'approche géographique consiste à juger un attrait selon ses qualités naturelles et culturelles. Cette acception déterministe de l'attractivité priorise les explications de l'attractivité par des acquis historiques et par des avantages de localisation. Des classifications telles que celle menée par Defert (1972) essaient d'affiner une analyse parfois jugée simpliste. Ainsi, un site deviendrait touristique à condition de rassembler des caractères remarquables pour son hydrôme (l'eau), son lithôme (les monuments et vestiges), son anthropôme (les activités humaines) ou son phytôme (la nature). Bien que discutables, ces critères permettraient une explication rapide de l'attractivité des petites et moyennes villes québécoises. Dans le cas de Rivière-du-Loup, le fleuve Saint-Laurent (hydrôme), les littoraux naturels (phytôme) et le centre-ville (anthropôme) expliquent l'intérêt de ce territoire pour les touristes.

Le développement touristique des périphéries s'inscrit dans la transformation du monde rural. Le secteur touristique symbolise une partie de la recomposition économique des régions et, dans un même temps, met en valeur les nouvelles composantes de la ruralité. Aujourd'hui, l'accès facile à la nature, les beaux paysages, la minimisation du stress au quotidien ou encore l'accès facile à la propriété incarnent les territoires ruraux. L'agriculture est encore importante, mais ne se situe plus au cœur des représentations contemporaines de la ruralité (Jean, 2012).

Les PMV de périphérie telles que Rivière-du-Loup mettent donc en valeur leurs richesses naturelles, agraires et paysagères à des fins touristiques. Toutefois, ces composantes de la ruralité s'avèrent sensibles aux changements climatiques.

Ainsi, dans le Bas-Saint-Laurent, les impacts des changements climatiques se concentrent surtout sur les littoraux (Ouranos, 2015). En effet, à l'augmentation des températures moyennes et au changement des régimes de précipitations, s'ajoutent les enjeux de diminution du pied de glace et d'augmentation du niveau du Saint-Laurent. Cela mène à une accélération de l'érosion dans certains secteurs, à la vulnérabilisation de certains littoraux urbanisés lors de tempêtes hivernales et à des risques de submersion. Les effets des changements climatiques peuvent aussi transformer les écosystèmes côtiers, argument touristique important dans la région.

Le système touristique de Rivière-du-Loup, qui mise beaucoup sur des attraits littoraux naturels et agricoles, devrait donc faire face à des transformations environnementales au cours des prochaines années. Nous voulons relever comment les acteurs perçoivent ces changements, mais aussi essayer de les outiller pour l'adaptation. Pour mieux saisir l'intégration de la question des changements climatiques dans le développement touristique de Rivière-du-Loup, plusieurs démarches méthodologiques ont été utilisées.

Dans un premier temps, une démarche de type recherche ouverte en innovation (living lab) a été lancée de janvier 2016 à mai 2019. Il s'agit d'un processus d'innovation ouverte avec une visée de recherche-action :

L'innovation ouverte consiste à accroître et à améliorer la collaboration avec les parties prenantes d'un écosystème et à impliquer les utilisateurs dans le processus de co-création. L'approche Living Lab vise à promouvoir et structurer la collaboration des parties prenantes, et sa caractéristique principale est que les utilisateurs deviennent des parties prenantes à part entière dans le processus de co-création et sont impliqués dès le début, dans un environnement réel (Lapointe et Guimont, 2015, p. 28) ${ }^{1}$. 
Dans notre cas, des acteurs touristiques, municipaux, privés ou des citoyens sont conviés à participer à des séances de travail collectif et d'expérimentation sur le sujet de l'adaptation aux changements climatiques du tourisme. Nous avons organisé cinq ateliers. Les deux premiers ateliers, tenus en 2016, rassemblaient environ 20 acteurs à l'échelle de la MRC. Ils ont mené à une journée d'intervention et de sensibilisation.

Pour la deuxième phase de ce living lab, nous avons ciblé le territoire d'expérimentation en collaborant de manière plus étroite avec trois acteurs : la direction du parc côtier, un organisme environnemental de protection du Saint-Laurent et la MRC. Le parc côtier Kiskotuk a alors été défini comme l'espace touristique sur lequel réfléchir à l'adaptation aux changements climatiques. Situé le long du fleuve Saint-Laurent, ce parc régional linéaire s'étend sur deux municipalités : Cacouna et L'Isle-Verte. Il englobe aussi l'île de Notre-Dame-des-SeptDouleurs. Plusieurs usages se côtoient et, parfois, se superposent ou s'opposent. La vocation résidentielle cohabite avec des usages agricoles et récréatifs. Les rôles de conservation et de réserve faunique occupent aussi une place importante. Le parc est à la fois un lieu de promenade pour les habitants et un pôle récréatif et touristique de la MRC.

Avant d'organiser les deux derniers ateliers, nous avons mené sept entretiens empathiques auprès de différents usagers du parc pour mieux comprendre les valeurs, les usages et les vulnérabilités de ce territoire. Finalement, 19 entretiens complémentaires ont été réalisés avec d'autres acteurs touristiques et municipaux de Rivière-du-Loup. Ils sont en cours d'analyse, mais certaines informations qu'ils contiennent sont intégrées à la présente réflexion.

\section{Résultats préliminaires}

Nous présentons donc les principaux enjeux de l'adaptation soulevés par les acteurs liés au tourisme de la MRC de Rivière-du-Loup lors de notre démarche. Parmi toutes les données collectées, nous utilisons surtout les sept entretiens empathiques menés au parc côtier Kiskotuk. En effet, certains passages révèlent bien des leviers d'action potentiels pour l'adaptation.

Dans les canevas de ces sept entretiens, nous ne posions pas de questions directement sur les changements climatiques. Nous voulions voir quels acteurs notaient des changements dans l'environnement du parc que nous pourrions associer aux changements climatiques. Cette démarche avait été suggérée par les acteurs présents au troisième atelier afin d'alimenter le contenu des deux dernières rencontres.

Tout d'abord, il apparait que seuls les acteurs en contact très fréquent avec le parc notent des changements (chasseurs, agriculteurs et intervenants touristiques).

Qu'est-ce que j'trouve qui a changé c'est les marées. L'eau a monte din champs asteur. Quand on cultive... avant ça, quand on cultivait y avait jamais d'eau qui montait din champs. Pis là asteur, quand on sème des plantes su'l bord des fossés, y-y pousse pas. Y pousse... pis là, quand la marée monte, ben là, c'est de l'eau salée. Faque là, à un moment donné (sourire)... Ça a d'la misère, ça l'aime pas ça. Tu vois ça reste, ça reste sur l'argile, sur le bord. Pis ça on - c'est un phénomène qu'on voyait pas beaucoup. Moi, quand $j$ 'tais ti-gars, on voyait jamais, jamais ça. Asteur, on en voit beaucoup là. Toutes... dans toutes les grandes marées là, asteur y a de l'eau dans l'chemin. (Entrevue 4)

Dans ce passage, cet agriculteur observe la submersion récurrente d'une partie de ses champs. Il compare cet événement à une époque où cela n'arrivait pas, selon son vécu. Ces inondations d'eau salée touchent son activité, car elles détruisent une partie de ses plants. Cela dit, il nous explique tout de même, plus tard dans l'entretien, que ces pertes sont résiduelles par rapport à la taille de son exploitation. Il réfléchit même à certaines potentialités qu'il pourrait tirer de la situation (culture de plantes pouvant pousser dans des sols salés).

Si l'on peut associer ces épisodes de submersion à la montée du niveau des eaux, l'érosion côtière s'avère aussi un facteur aggravant. Un chasseur rend compte de ce phénomène naturel, accéléré par les changements climatiques (Ouranos, 2015) :

Faque l'érosion... eh, on voit que ça s'effrite. Eh, la mer monte de plus en plus...... dans le sens la partie de la batture versus la partie vaseuse re-gagne du terrain là (...) Dans les 40 ans où j’y ai été là...Ça a changé beaucoup, beaucoup, beaucoup, beaucoup. Il y a 
beaucoup plus de marais qu'on avait, mais s'parce que là c'est la mer qui vient les chercher. Faque, même, j'ai une cache qui est sur le bord du-de la rivière, elle est à veille de s'en aller là. Parce que là... Elle est rendue... Avant y avait peut-être facilement la longueur de mon bureau devant, en battures. Maintenant, c'est la rivière qui commence là. (Entrevue 1)

Ici encore, cet utilisateur de longue date du territoire insiste sur une évolution du territoire par rapport à quelques décennies auparavant. Le phénomène mis en avant est la transformation des formes de la côte sous l'effet de l'érosion côtière. Cette érosion est accélérée par la diminution progressive du pied de glace, comme le relève un intervenant touristique de l'île de Notre-Damedes-Sept-Douleurs (L'Isle-Verte) :

Sur la rive nord de l'île - en particulier là, les tempêtes maintenant là eh, bouleversent la plage eh, les glaçons viennent ravager comme si une tempête d'hiver, comme la glace est toute fragmentée, plus d'un seul tenant maintenant. Les glaçons se promènent et endommagent le couvert végétal de plus en plus loin maintenant. Alors ça, c'est des changements que j'ai remarqué, moi, parce qu'avant eh, avant, on n'avait pas ça, j'veux dire. Avant, la glace prenait et y avait un espèce de parement de glace solide là et c'était très rare qu'on voyait les glaçons éparpillés, montés sur les plages, comme j'l'ai vu y a 2 ans. Comme j'l'ai vu l'année dernière, même. (Entrevue 5)

Cet interlocuteur nous décrit très précisément et très visuellement les impacts de la diminution de l'englacement de l'estuaire. Cela aggrave un phénomène vécu en aval de l'île, sur le littoral continental du parc côtier :

Toute l'érosion... On le vit. Les marais se brisent, se... changent, entre autres, il y a une cache ou s'qu'on, une des caches, puisqu'on en a plusieurs, devant la cache avant ce n'était pas... un marais (rires). Maintenant, c'est rendu (sourire) : un grand marais. Il est rendu tellement grand qu'on peut le voir sur Google Map. (Entrevue 1)
La transformation du paysage s'observe donc déjà. Pourtant, les acteurs rencontrés ne mentionnent pas directement les changements climatiques, mais signalent l'accélération de l'érosion côtière, la diminution du pied de glace et la submersion. Dans leur pratique du territoire, ils font face à ces changements. L'agriculteur constate la submersion à un niveau qu'il n'avait jamais vu auparavant. Le chasseur constate qu'une de ses caches pour la chasse est désormais inutilisable à cause de l'érosion. Le responsable d'un attrait touristique de Notre-Dame-des-Sept-Douleurs (L'Isle-Verte) note la diminution du pied de glace et les glaçons sur la rive nord de l'île.

Ce projet de recherche s'appuie aussi sur la thèse de doctorat en études urbaines d'Alexis Guillemard, au cours de laquelle 19 entretiens ont été réalisés dans la MRC de Rivière-du-Loup et dans la MRC de Rimouski-Neigette auprès d'intervenants touristiques, d'acteurs de l'aménagement du territoire et d'acteurs environnementaux. Si nous ne pouvons pas encore détailler le contenu des verbatims non codés, plusieurs éléments en ressortent tout de même. Pour l'instant, tous les acteurs rencontrés partagent une conscience et une inquiétude par rapport aux changements climatiques. Nous n'avons pas encore rencontré d'acteurs qui émettent des doutes sur leurs occurrences. Toutefois, face au défi, le sentiment d'impuissance est partagé par tous les acteurs. Ce sentiment d'impuissance fait écho à celui relevé par ceux qui ont participé aux ateliers du living lab.

Seuls 2 acteurs parmi les 19 répondants relèvent avec certitude des transformations physiques du territoire et s'inquiètent de l'érosion. Les autres participants émettent des hypothèses et associent certains événements météorologiques aux changements climatiques, mais insistent sur l'incertitude de leurs constats. Le manque de connaissance et le sentiment d'impuissance poussent les acteurs rencontrés à nous avouer peu agir pour l'adaptation dans leur sphère professionnelle. Individuellement, dans leur vie personnelle, tous essaient de faire des efforts. En revanche, dans le contexte d'une organisation professionnelle, ils ne trouvent pas ou peu de ressorts à activer.

Enfin, pour les enjeux spécifiques aux petites villes, plusieurs acteurs signalent que des organi- 
sations aux équipes réduites sont souvent complètement monopolisées par les enjeux du quotidien. Selon ces répondants, cette échelle ne dispose pas de ressources suffisantes (en temps, en employés, en argent) pour affronter les enjeux à long terme, comme ceux des changements climatiques. De plus, certaines perceptions pointent du doigt les élus et leur manque de vision à moyen ou long terme. Les périodes électorales, trop courtes, ne seraient pas adaptées à la territorialisation d'une adaptation aux changements climatiques.

\section{Enjeux pour la production d'espaces touristiques littoraux adaptés}

Les différentes phases de la recherche menée de 2016 à 2019 révèlent chacune un enjeu principal.

\subsection{La sensibilisation territorialisée}

La première phase correspond aux ateliers menés en démarche living lab et au prototypage qui a suivi. Les acteurs touristiques mettent en avant la nécessité de la sensibilisation. Toutefois, il ne s'agit pas de cours ni d'informations générales sur les changements climatiques, mais d'une sensibilisation territorialisée qui pourrait toucher les visiteurs, les entreprises touristiques et les résidents.

Dans un environnement en transformation, pour durabiliser la production d'espaces touristiques littoraux, il semble donc nécessaire de concevoir des outils (matériels ou éducationnels) qui permettent de réaliser l'ampleur des changements à venir. De plus, dans une PMV, cette sensibilisation ne peut être cloisonnée au tourisme. Elle devrait aussi bénéficier aux populations et/ou à d'autres secteurs économiques.

Si on la lie au prisme de la théorie de la production de l'espace, une sensibilisation territorialisée permet de donner au territoire des capacités immatérielles à lancer l'adaptation. En effet, l'espace des représentations joue un rôle fondamental dans l'action face aux changements climatiques. Ainsi, l'accès à l'information et aux connaissances des effets des changements climatiques permet de savoir à quel niveau agir. Parallèlement, la construction de symboles peut construire un imaginaire du risque ou une conscience des changements climatiques et de leurs effets.

Revue Organisations \& Territoires $•$ Volume $29 \cdot \mathrm{N}^{\circ} \mathbf{1} \cdot 2020$

\subsection{La mobilisation et la mise en valeur des savoirs vernaculaires}

Les résultats du travail sur le parc côtier Kiskotuk nous donnent aussi des enseignements intéressants. Ce territoire est utilisé quotidiennement par des acteurs qui en exploitent les richesses (agricoles, fauniques et touristiques). Ces personnes possèdent des savoirs vernaculaires accumulés par une pratique assidue du milieu :

Les savoirs géographiques vernaculaires sont $[\ldots .$.$] un engagement de tous les sens, qui les$ fonde dans une expérience ontologique de l'espace et du milieu. Ils sont nécessairement subjectifs et contextualisés, et ne peuvent donc être que pluriels. Si l'on peut repérer des caractères communs à tout savoir géographique vernaculaire, les contenus sont toujours différents, comme les cultures dont ils sont l'expression (Collignon, 2005, p. 325).

Les changements climatiques au Québec sont pour le moment - une réalité assez lointaine, avec peu d'impacts visibles sur les territoires. Toutefois, dans une société tertiarisée, nomade et urbaine, les individus capables de constater les transformations physiques de leur milieu de vie sont rares. La pratique répétée, hebdomadaire et ancienne (donc l'expérience) d'un milieu donne des référentiels physiques et temporels permettant une comparaison. Dans le cas de la MRC de Rivière-du-Loup, les agriculteurs, les chasseurs et les usagers fréquents du territoire ont été en mesure de relever les changements. La valorisation de ces savoirs vernaculaires peut permettre la production d'espaces touristiques adaptés aux changements climatiques.

Les PMV périphériques bénéficient d'acteurs capables de constater des effets des changements climatiques. Alors que ces individus relèvent des impacts locaux, ils ne les intègrent pas forcément dans une évolution globale. Il serait pourtant pertinent de mobiliser leurs observations et leurs connaissances dans des programmes d'adaptation, qui peinent parfois à s'ancrer dans les territoires.

\subsection{La mobilisation des ressources}

Lors de ce projet de recherche dans une PMV, il nous a semblé que les ressources internes au territoire peuvent être mobilisées plus facilement que dans un contexte métropolitain. Les petites 
villes, par leur échelle, auraient une capacité de mobilisation plus transversale et intersectorielle que dans une métropole. Cependant, trouver un relai sur le territoire s'est avéré précieux. Dans notre cas, le Living Lab en innovation ouverte (LLio) du Cégep de Rivière-du-Loup a été l'organisation de relais entre les chercheurs et les acteurs, puis entre les différents secteurs d'activité.

Malgré les initiatives d'innovation ouverte et les enseignements que nous en avons tirés, il reste toutefois plusieurs obstacles à l'adaptation, notamment en ce qui concerne l'accès à des ressources externes au territoire. Lors des ateliers, le manque d'outils ainsi que le manque de moyens humains et financiers ont été souvent désignés comme des limites importantes à l'action. Si des ressources provinciales (Action-Climat Québec et le programme Climat municipalités) et fédérales (Fonds d'action pour le climat, programmes de soutien de la Fédération canadienne des municipalités) existent, leur mobilisation se heurte aux réalités d'une échelle municipale dont les équipes réduites sont accaparées par les enjeux du quotidien.

Outre les limites inhérentes à la structure municipale d'une petite ville, les acteurs municipaux ne disposent pas toujours de connaissances ni de moyens techniques pour innover les réponses aux changements climatiques. Ainsi, certaines stratégies d'immunisation sont privilégiées par rapport à des solutions plus longues à mettre en œuvre. Par exemple, l'enrochement constitue la réponse récurrente aux enjeux de submersion ou d'érosion sur les rivages du Saint-Laurent, alors que la voie du génie végétal ne se développe que dans des secteurs très localisés.

\section{Conclusion}

Les petites villes de périphérie comme Rivière-duLoup, selon nous, font aujourd'hui partie de la ruralité contemporaine. On y retrouve la multifonctionnalité de l'espace rural, déclinée « en quatre fonctions souvent concurrentes: productive, résidentielle, récréative et de préservation » (Simard, 2017, p. 113). En outre, plusieurs représentations de la ruralité, telles que la nature, l'agriculture et une certaine qualité de vie (Jean, 2012) sont mises en valeur par le tourisme. À Rivière-du-Loup, certains de ces attraits, surtout quand ils sont en position littorale, sont déjà touchés par les effets des changements climatiques et peuvent subir une intensification de ces transformations. Déconstruire l'attractivité de ces lieux révèle la grande diversité des richesses territoriales affectées (ou potentiellement affectées) par les changements climatiques : paysage, biodiversité, agriculture, patrimoine, etc.

Par conséquent, réfléchir à l'adaptation du tourisme aux changements climatiques et l'expérimenter dépassent le seul intérêt des acteurs touristiques, et peuvent aussi bénéficier à d'autres composantes du territoire. À plusieurs reprises, les acteurs ont insisté sur les externalités positives d'initiatives initialement appliquées au tourisme pour l'ensemble de la collectivité. Par exemple, une offre expérientielle de sensibilisation menée dans le parc côtier Kiskotuk bénéficierait aussi aux habitants qui le fréquentent. Toutefois, la dialectique entre tourisme et communauté reste présente, ouvrant la porte au risque d'une adaptation ciblée sur la fréquentation touristique, au détriment des représentations et usages locaux.

Ainsi, la MRC de Rivière-du-Loup, territoire hybride, s'avère un bon laboratoire pour des solutions innovantes, à la fois utiles pour des grands centres urbains, mais aussi pour les espaces ruraux ayant moins de services. La démarche en mode living lab montre l'importance des relais territorialisés pour innover et trouver des options d'adaptation aux changements climatiques, elles-mêmes adaptées au contexte local.

Trois enjeux se superposent. Le premier, révélé par la phase des ateliers, est de sensibiliser les acteurs territoriaux aux effets locaux des changements climatiques. Un autre enjeu revient à trouver des personnes qui, par leurs connaissances et leur pratique du territoire, constatent des changements. Les savoirs de ces informateurs clés doivent absolument être valorisés pour trouver des moyens de concrétiser l'adaptation, car ils sont une traduction de l'espace perçu comme en transformation. Enfin, l'enjeu de donner des outils et des moyens à une échelle qui en manque par rapport aux métropoles s'avère pertinent pour dépasser un cadrage cognitif des changements climatiques, où la globalité du phénomène, et ses temporalités, et la proximité des impacts laissent un vide à l'échelle méso pour territorialiser l'adaptation. 


\section{NOTES}

1 Traduction des auteurs de : «Open innovation is about increasing and improving collaboration with an ecosystem's stakeholders and engaging users in the co-creation process. The living lab approach aims to promote and structure stakeholder collaboration, and its key feature is that users become fully fledged stakeholders in the co-creation process and are involved from the very beginning, in a real-life environment.

\section{RÉFÉRENCES}

Collignon, B. (2005). Que sait-on des savoirs géographiques vernaculaires? Bulletin de l'Association de géographes français, 82(3), 321-331. Repéré à www.persee.fr/doc/bagf_0004-5322_2005_num_82_3_2467

Defert, P. (1972). Les ressources et les activités touristiques : essai d’intégration. Cabiers du CET, série C, 19. Marseille : Université d'Aix-Marseille.

Gagnon, S. (2003). L'écbiquier touristique québécois. Sainte-Foy, QC : Presses de l’Université du Québec.

Kadri, B. et Pilette, D. (2017). Le tourisme métropolitain renowvelé. Sainte-Foy, QC : Presses de l'Université du Québec.

Jamot, C. (2003). Les petites villes et la dynamique touristique : l'exemple de La Souterraine (Creuse). Dans L'avenir des petites villes, Actes du Colloque international de Clermont-Ferrand (p. 141-154). Clermont-Ferrand, France: Presses universitaires Blaise Pascal.

Jean, S. (2012). Les représentations sociales de la ruralité et l'urbanité québécoise contemporaine : une approche par la cartographie conceptuelle. Recherches sociographiques, 53(1), 103-131. https://doi.org/10.7202/1008921ar

Judd, D. (1999). Constructing the tourist bubble. In D. Judd \& S. Fainstein (Eds.), The tourist city (p. 35-53). New Haven, CT: Yale University Press.

Lapointe, D., \& Guimont, D. (2015). Open innovation practices adopted by private stakeholders: Perspectives for living labs. Info, 17(4), 67-80. https://doi.org/10.1108/info-01-2015-0003

Lefebvre, H. (1974/2000). La production de l'espace (4e éd.). Paris : Anthropos.

Ouranos (2015). Vers l'adaptation: synthèse des connaissances sur les changements climatiques au Québec. Montréal, QC : Ouranos. Repéré à www.ouranos.ca/synthese-2015

Pilette, D. et Kadri, B. (2005). Le tourisme métropolitain : le cas de Montréal. Sainte-Foy, QC : Presses de l’Université du Québec.

Pumain, D. (1999). Quel rôle pour les villes petites et moyennes des régions périphériques? Revue de géographie alpine, 87(2), 167-184. Repéré à www.persee.fr/doc/rga_0035-1121_1999_num_87_2_2950

Simard, M. (2017). Environnement, conflits et tractations entre divers acteurs ruraux au Québec : une mobilisation inégale du capital environnemental? Norois, 243(2), 111-126. Repéré à www.cairn.info/revue-norois-2017-2-page-111.htm

Statistique Canada (2016a) Profil du recensement, Recensement de 2016 - Rivière-du-Loup, Ville [Subdivision de recensement], Québec et Québec [Province]. Repéré à https:/ /www12.statcan.gc.ca/census-recensement/2016/dp-pd/prof/ details $/$ page.cfm?Lang $=$ F\&Geo1 $=$ CSD $\&$ Code $1=2412072 \& G e o 2=$ PR\&Code2 $=24 \&$ Data $=$ Count\&SearchText $=$ Riviere-duLoup\&SearchType $=$ Begins $\&$ SearchPR $=01 \& B 1=$ All $\&$ TABID $=1$

Statistique Canada (2016b). Profil du recensement, Recensement de 2016 - Rivière-du-Loup, Municipalité régionale de comté [Division de recensement], Québec et Québec [Province]. Repéré à https://www12.statcan.gc.ca/census-recensement/2016/dp$\mathrm{pd} /$ prof/details/page.cfm?Lang $=\mathrm{F} \& \mathrm{Geo} 1=\mathrm{CD} \&$ Code $1=2412 \& \mathrm{Geo} 2=\mathrm{PR} \&$ Code2 $=24 \&$ Data $=$ Count $\&$ SearchText $=$ Riviere-duLoup\&SearchType $=$ Begins $\&$ SearchPR $=01 \& B 1=$ All 